\title{
Effectiveness and Key Success Factors for Implementation of Problem-Based Learning in Debre Tabor University: A Mixed Methods Study
}

\author{
Awoke Wondie ${ }^{*}$, Tegbar Yigzaw ${ }^{2}$, Solomon Worku
}

\section{OPEN ACCESS}

Citation: Awoke Wondie, Tegbar Yigzaw, Solomon Worku. Effectiveness and Key Success Factors for Implementation of Problem-Based Learning in Debre Tabor University: A Mixed Methods Study. Ethiop J Health Sci. 2020;30(5):803.doi:http:// dx.doi.org/ 10.4314/ejhs.v30i5.21

Received: December 13, 2019

Accepted: May 4, 2020

Published: September 1, 2020

Copyright: (C) 2020 Awoke Wondie, et al. This is an open access article distributed under the terms of the Creative Commons Attribution License, which permits unrestricted use, distribution, and reproduction in any medium, provided the original author and source are credited.

Funding: Nil

Competing Interests: The authors declare that this manuscript was approved by all authors in its form and that no competing interest exists.

Affiliation and Correspondence:

${ }^{1}$ Reproductiive Health, Debretabor University, Amhara Regional State, Ethiopia

${ }^{2}$ JHPIEGO, Resources for Health, Addis Ababa, Ethiopia

${ }^{3}$ Human Resources Directorate, Federal Ministry of Health, Addis Ababa, Ethiopia

*Email: awokegiletew@yahoo.com

\begin{abstract}
BACKGROUND: Problem-based learning has been adopted as a core educational strategy for education of health professionals in more than a dozen of higher education institutions in Ethiopia. Debre Tabor University College of Health Sciences (DTUCHS) is one of the adopters. However, its effectiveness has not been researched yet. Thus, the objective of this study is to assess the quality of PBL implementation, its effectiveness in developing desired student learning outcomes and factors that facilitate or impede PBL implementation.
\end{abstract}

METHODS: A cross-sectional study was conducted in DTUCHS from May to June 2018. We collected quantitative data from students and tutors using self-administered questionnaire. We complemented this with key informant interviews with academic leaders. We computed descriptive statistics from quantitative data while qualitative data were subjected to thematic analysis.

RESULTS: A total of 308 students, 42 tutors and 8 academic leaders were included in the study. Students, tutors and academic leaders perceived that PBL was effective in developing knowledge, problem-solving skills, self-directed learning skills and collaboration competencies. The implementation process showed the existence of clear objectives, appropriate cases, and reasonable workload. Students rated tutors' performance positively, and tutors also rated student learning affirmatively. However, unlike tutors, students thought that the assessment of student performance in $P B L$ was not appropriate. The factors that facilitated PBL implementation were students' and tutors' buy-in, clear curriculum design, adequate infrastructure, commitment to hire more faculty and develop their teaching skills continuously and strong coordination and monitoring.

CONCLUSION: The findingsof our study support the introduction of $P B L$ in a resource-constrained setting. Students, tutors and academic leaders perceived PBL to be effective in achieving desired student learning outcomes. Its implementation was considered consistent with the principles of PBL. Respondents identified the presence of enabling factors to implement PBL in Debre Tabor University (DTU).

KEYWORDS: Problem-based learning, Implementation, Process, Perception, Ethiopia 


\section{INTRODUCTION}

Problem-based learning (PBL) is an educational strategy in which "problems are used as a trigger for learning; students collaborate in small groups; learning takes place under the guidance of a tutor; learning is student-initiated; and the curriculum includes ample time for self-study" (1). PBL curriculum was pioneered by the Faculty of Medicine at McMaster University in 1968 due to dissatisfaction with the observation that medical students did not retain and apply the basic science knowledge by the time they enter clerkship. PBL has since spread throughout the world transcending disciplines and the primarysecondary-tertiary education divide (2).

Proponents of PBL argue that it facilitates comprehension and long-term retention of knowledge. Problems and small group discussions in PBL encourage activation and elaboration of prior knowledge which increases motivation to learn because problems arouse situational interest (1). However, the effectiveness of PBL compared to lecture-based curriculum in improving learning outcomes has been a subject of several studies and debates (3). A recent systematic review of the effects of PBL on physician competence after graduation found that physicians trained through PBL were better at coping with uncertainty, appreciating legal and ethical aspects of healthcare, communication skills and continuing self-directed learning than those trained with traditional education strategy. However, the results were inconclusive when it came to knowledge outcomes, indicating that PBL is not a panacea (4). Some argue that the lack of efficacy of PBL is due to the varied understanding and practice of the method in different contexts (2). Importantly though, there is a growing realization that combining PBL with other teaching/learning methods may yield better results (3).

Despite the rapid acceptance, many attempts to use PBL have failed to reach the results expected. Apart from philosophical objections as an educational method and resistance from faculty, PBL implementation could be affected by many factors that could emanate from the curriculum, institution and students. Ignoring these factors was the major reason for failed PBL implementation in many institutions $(5,6)$.
PBL as a curriculum concept has a relatively short history in Ethiopia but is becoming increasingly important. PBL was adopted as an educational strategy in the 2011 innovative medical curriculum, which is being implemented in 13 medical schools around the country (7). The College of Health Sciences at Debre Tabor University (DTUCHS) also adopted PBL as one of the key educational strategies when it designed innovative curricula for medicine and midwifery in 2013 as well as other disciplines in subsequent years (8). PBL is also integrated into recently developed national curricula for different health disciplines, indicating a trend towards more widespread adoption in Ethiopia. Given its growing importance in health science curricula, it is high time that a study is done to evaluate whether PBL results in better learning outcomes. Moreover, although context has an important impact on the success or failure of PBL (2), we have not come across a local study on PBL implementation. Therefore, this study aimed to assess the quality of PBL implementation, its effectiveness in developing desired student learning outcomes and the factors that facilitate or impede PBL implementation.

\section{METHODS}

Study design and period: A cross-sectional study that combines a self-administered questionnaire and qualitative interviews was conducted between May and June 2018.

Study setting: The study was done in Debre Tabor University College of Health Sciences (DTUCHS). DTUCHS designed and implemented innovative curricula that combines PBL along with other contemporary teaching/learning methods. The curricula suggest that a group of 5 to 8 students meet twice per week to complete one PBL case in a week. In the first meeting, learners identify problems, generate hypotheses and explain mechanisms with gradual disclosure of information. Days between the two sessions are dedicated to selfstudy on learning issues identified. During the second meeting, students discuss the learning issues to apply what they have learned to the problem and synthesize the learning agendas addressed. Faculty from clinical, biomedical and public health disciplines develop and/or review 
PBL cases before the next academic year begins. This study focused on departments of medicine and midwifery that have a longer history of PBL implementation than the others. Both medical and midwifery students have PBL sessions every week starting from the second semester of the first year through the final year.

Population and sampling: The study populations for the self-administered questionnaire were students and tutors while we targeted academic leaders for the key informant interviews. At the time of the study, there were 220 medical students from year one through year five and 88 midwifery students in second and third years. However, first year midwifery students had not started implementing PBL during data collection and were thus excluded from this study.

At the time of data collection, there were 30 academic staff in the Department of Medicine and 26 in the Department of Midwifery, and we sought to cover all those who met the inclusion criteria. The inclusion criteria for academic staff were participating in PBL case development and/or tutoring for at least one academic year. The college had 13 academic leadership positions at the time of the study. Academic leaders (deans, department heads and coordinators) with experience of 12 months or more were purposively selected for the qualitative interviews. Qualitative interviews continued until a point of saturation was reached.

\section{Data Collection}

Quantitative data: Self-administered questionnaire was used to obtain students' and tutors' perspectives on effectiveness of PBL (17 items arranged in 6 main themes), process of PBL implementation (14 items for students and 13 items for tutors, both arranged in 5 main themes), student performance (17 items arranged in 6 main themes), tutor performance (13 items arranged in 5 main themes) and barriers for PBL implementation ( 9 items for students and 8 items for tutors). The response options for the first four variables were a 5-point Likert scale: $5=$ strongly agree, $4=$ agree, $3=$ neutral, $2=$ disagree, and $1=$ strongly disagree. The response options for the barriers of implementation were a four-point Likert scale: $1=$ not a barrier, $2=$ somewhat a barrier, $3=$ moderate barrier and $4=$ extreme barrier. The items in the questionnaire were adapted from previous studies (9-14).

Qualitative data: Semi-structured interviews were conducted to capture the views of academic leaders regarding the effectiveness of PBL, its implementation and the factors that facilitate or impede PBL implementation. The interview was tape-recorded and completed within an average of 25 minutes. The lead author conducted all the interviews.

Validity and reliability: The data collection tools for tutors, students and academic leaders were pilot-tested with 9 tutors, 11 students and 3 academic leaders, respectively. Participants for the pilot-test were not from the departments of medicine and midwifery. Appropriate modifications were made to the tools following the pilot, which included writing more clear instructions, editing some items and removing items that were not aligned with the objectives of the study. The reliability of quantitative tools was estimated using the Cronbach's coefficient alpha test, which yielded $96 \%$ and $91 \%$ for tutors and students, respectively, showing a very high level of internal consistency.

Data analysis: The quantitative data were entered and processed using Statistical Package for Social Sciences (SPSS), version 20, to calculate percentage, median and inter-quartile range for each item and the main factors. We merged "Strongly agree" and "Agree" in the narrative for ease of readability. Perceived barriers of implementation were dichotomized and presented as "Barrier" (by merging $1=$ Not a barrier and $2=$ Somewhat a barrier), and "Major barrier" (by merging $3=$ Moderate barrier, and 4 $=$ Extreme barrier).

The qualitative interviews were transcribed verbatim by the lead author. Braun and Clarke's six-phase framework for doing a thematic analysis was applied, which includes familiarizing with data, generating initial codes, searching for themes, reviewing themes and defining themes (15). The evaluator coded and categorized the responses, and several themes emerged from them. The evaluator reviewed the interviews again and a point of saturation was ensured in the $8^{\text {th }}$ interview coding when the same 
themes and sub-themes were identified. The final result was presented by triangulating the quantitative data in a narrative way supported by selected direct quotes.

Ethics: This study was approved by Debre Tabor University Research Ethics Committee. All participants were informed of the objective of the study and gave their written consent to participate.

\section{RESULTS}

Characteristics of participants: A total of 308 students (220 Medicine and 88 Midwifery) and 42 tutors (18 Medicine and 24 Midwifery) returned a completed questionnaire, which yielded a response rate of $100 \%$. The mean age of students was $20.97 \pm 2.07$ years while that of tutors was $27.02 \pm 3.17$ years (Table 1$)$.

Table 1: Characteristics of quantitative study participants among the departments of medicine and midwifery, DTU, 2018.

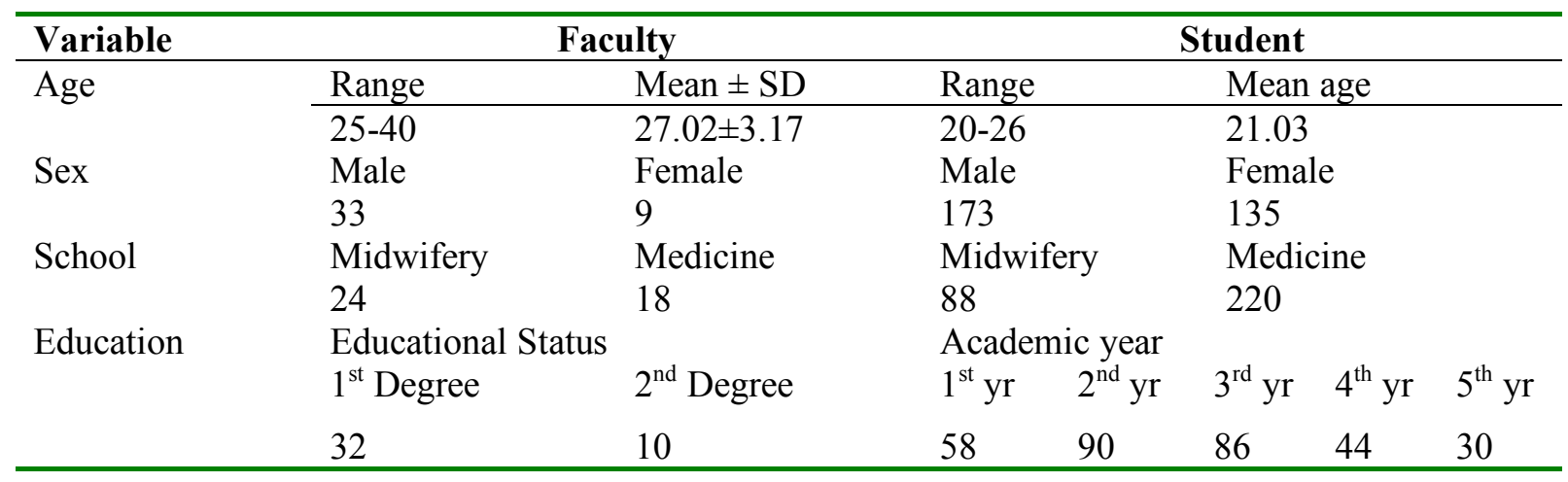

Perceived effectiveness of PB: Students and tutors agreed that PBL facilitated attainment of desired learning outcomes: developing knowledge, problem-solving skills, self-directed learning skills, motivation and group collaboration skills. Promoting group collaboration, improving motivation and constructing professional knowledge were the top three benefits, drawing agreement from $79.3 \%$, $76.9 \%$, and $75.5 \%$ of students, respectively. Over half of students $(57.3 \%)$ also said they preferred PBL over lecture(Table 2). Tutors' perceptions of PBL's educational benefits were even more favourable, with the top three ratings going to promoting group collaboration (95.1\%), improving motivation (92.8\%) and developing self-directed learning skills (86.3\%). Most tutors $(73.8 \%)$ also said they preferred PBL over lecture (Table 3). The perceived effectiveness of PBL in student learning was also echoed in the qualitative component of the study. Academic leaders reported that realistic scenarios help students to relate concepts to everyday activities, and discussion among them strengthens understanding of subject matter. The following quote from the head of Medicine Department goes:

'PBL enables them to remember what they understand from cases and share what they understand, improving their communication and reasoning skills. So, it was effective in supporting student learning.'

Almost all interviewed leaders concluded that PBL is their preferred teaching method. A biomedical unit coordinator said:

"I prefer PBL over other teaching methods in facilitating student learning effectively. Compared to lecture method, I think PBL is the best teaching method."

PBL implementation process: Most students $(66.2 \%)$ considered the workload imposed by PBL was reasonable, and about $65.4 \%$ of the students reported that PBL cases were appropriate and adjusted to students' level of learning. However, only $51.6 \%$ of them reported the existence of clear expectations or what to do in PBL sessions. 
Table 2: Students' perception of PBL as a teaching strategy in the departments of medicine and midwifery, DTU, $2018(\mathrm{~N}=308)$.

\begin{tabular}{|c|c|c|c|c|c|c|c|}
\hline S.no & Item & $\begin{array}{l}\text { SDA } \\
\text { n(\%) } \\
\end{array}$ & $\begin{array}{c}\text { DA } \\
\mathbf{n}(\%) \\
\end{array}$ & $\begin{array}{c}\mathbf{N} \\
\mathbf{n}(\%) \\
\end{array}$ & $\begin{array}{c}\mathbf{A} \\
\mathbf{n}(\%) \\
\end{array}$ & $\begin{array}{c}\mathbf{S A} \\
\mathbf{n}(\%) \\
\end{array}$ & $\begin{array}{c}\text { Mdn } \\
\text { (IQR) } \\
\end{array}$ \\
\hline \multicolumn{2}{|c|}{$\begin{array}{l}\text { Construction of Professional } \\
\text { knowledge }\end{array}$} & $12(3.9)$ & $24(7.8)$ & $39(12.5)$ & $123(39.7)$ & 111(35.8) & $4.00(1.50)$ \\
\hline 1 & $\begin{array}{l}\text { Use and activate my prior } \\
\text { knowledge }\end{array}$ & $13(4.2)$ & $21(6.8)$ & $29(9.4)$ & $134(43.4)$ & $111(35.9)$ & $4.00(1)$ \\
\hline 2 & $\begin{array}{l}\text { Interpret, analyze, and apply } \\
\text { key concepts precisely }\end{array}$ & 9.(2.9) & $19(6.1)$ & $37(12)$ & $146(47.2)$ & $97(31.4)$ & $4.00(1)$ \\
\hline 3 & $\begin{array}{l}\text { Enhance deep understanding } \\
\text { of knowledge }\end{array}$ & $8(2.6)$ & $28(9.1)$ & $38(12.3)$ & $123(39.8)$ & 111(35.9) & $4.00(1)$ \\
\hline 4 & $\begin{array}{l}\text { Gain and retain knowledge } \\
\text { for a long period }\end{array}$ & $19(6.2)$ & $29(9.4)$ & $50(16.2)$ & $87(28.2)$ & $123(40)$ & $4.00(2)$ \\
\hline \multicolumn{2}{|c|}{$\begin{array}{l}\text { Development of Problem- } \\
\text { solving skills }\end{array}$} & $15(4.9)$ & $30(9.6)$ & 53(17.2) & 141(45.7) & $69(22.4)$ & $4.00(1)$ \\
\hline 5 & $\begin{array}{l}\text { Increase my ability to solve } \\
\text { real-world problems }\end{array}$ & $\begin{array}{c}24 \\
(7.8)\end{array}$ & $48(15.5)$ & $66(21.4)$ & $137(44.3)$ & $33(10.7)$ & $4.00(1)$ \\
\hline 6 & $\begin{array}{l}\text { Encourage me to consider } \\
\text { alternatives when solving a } \\
\text { problem }\end{array}$ & $\begin{array}{c}10 \\
(3.2)\end{array}$ & $25(8.1)$ & $49(15.9)$ & $147(47.6)$ & $77(24.9)$ & $4.00(2)$ \\
\hline 7 & $\begin{array}{l}\text { Enable me to make } \\
\text { reasonable conclusions }\end{array}$ & $\begin{array}{c}12 \\
(3.9)\end{array}$ & $16(5.2)$ & $44(14.2)$ & $139(45)$ & $97(31.4)$ & $4.00(1)$ \\
\hline \multicolumn{2}{|c|}{$\begin{array}{l}\text { Development of Self-directed } \\
\text { learning }\end{array}$} & $19(6.1)$ & $32(10)$ & $51(16.7)$ & $124(40.1)$ & $82(26.5)$ & $4.00(1.50)$ \\
\hline 8 & $\begin{array}{l}\text { Identify gaps in my } \\
\text { knowledge }\end{array}$ & $22(7.1)$ & $25(8.1)$ & $45(14.6)$ & $118(38.2)$ & $98(31.7)$ & $4.00(2)$ \\
\hline 9 & $\begin{array}{l}\text { Identify and utilize } \\
\text { resources for PBL like a } \\
\text { reference, internet }\end{array}$ & $16(5.2)$ & $29(9.4)$ & $42(13.6)$ & $135(43.7)$ & $86(27.8)$ & $4.00(2)$ \\
\hline 10 & Think independently & $16(5.2)$ & $28(9.1)$ & $63(20.4)$ & $127(41.1)$ & $74(23.9)$ & $4.00(1)$ \\
\hline 11 & Plan my work & $22(7.1)$ & $46(14.9)$ & $56(18.1)$ & $115(37.2)$ & $69(22.3)$ & $4.00(1)$ \\
\hline \multicolumn{2}{|c|}{ Improvement of Motivation } & $19(6.1)$ & $23(7.6)$ & $29(9.4)$ & $114(37.0)$ & $123(39.9)$ & $4.00(1.50)$ \\
\hline 12 & Learn more & $16(5.2)$ & $19(6.1)$ & $24(7.8)$ & $121(39.2)$ & $128(41.4)$ & $4.00(1)$ \\
\hline 13 & $\begin{array}{l}\text { Stimulate my interest in } \\
\text { learning }\end{array}$ & $21(6.8)$ & $28(9.1)$ & $34(11)$ & $107(34.6)$ & $118(38.2)$ & $4.00(2)$ \\
\hline \multicolumn{2}{|c|}{ Promoting group collaboration } & 11(3.4) & $19(6.1)$ & $34(11)$ & $135(44)$ & $109(35.3)$ & $\begin{array}{c}4.00 \\
(1.00) \\
\end{array}$ \\
\hline 14 & $\begin{array}{l}\text { Improves my ability to } \\
\text { participate in open } \\
\text { discussion of differing } \\
\text { opinions }\end{array}$ & $9(2.9)$ & $19(6.1)$ & $37(12)$ & $138(44.7)$ & $105(34)$ & $4.00(1)$ \\
\hline 15 & $\begin{array}{l}\text { Share what I learned or } \\
\text { understand }\end{array}$ & $9(2.9)$ & $12(3.9)$ & $31(10)$ & $135(43.7)$ & $121(39.2)$ & $4.00(1)$ \\
\hline 16 & Respect others opinion & $14(4.5)$ & $26(8.4)$ & $34(11)$ & $134(43.4)$ & $100(32.4)$ & $4.00(1)$ \\
\hline $\begin{array}{l}\text { I pre } \\
\text { effec }\end{array}$ & $\begin{array}{l}\text { efer PBL to lecture as an } \\
\text { tive method of learning }\end{array}$ & 40(13) & $46(15)$ & $45(14.6)$ & $85(27.5)$ & $92(29.8)$ & $4.00(3.00)$ \\
\hline
\end{tabular}


Table 3: Tutors' perception of PBL as a teaching strategy in the departments of medicine and midwifery, DTU, $2018(\mathrm{~N}=42)$.

\begin{tabular}{|c|c|c|c|c|c|c|c|}
\hline S.no & Item & $\begin{array}{l}\text { SDA } \\
\text { n(\%) }\end{array}$ & $\begin{array}{c}\text { DA } \\
\text { n(\%) }\end{array}$ & $\begin{array}{c}\mathbf{N} \\
\mathbf{n}(\%)\end{array}$ & $\begin{array}{c}A \\
n(\%)\end{array}$ & $\begin{array}{c}\text { SA } \\
\text { n(\%) }\end{array}$ & $\begin{array}{c}\text { Mdn } \\
\text { (IQR) }\end{array}$ \\
\hline \multicolumn{2}{|c|}{ Construction of Professional knowledge } & 1.5(3.5) & $5(10.7)$ & 1(1.7) & 16(39.3) & 19(44.6) & $\begin{array}{l}4.25 \\
(1.00)\end{array}$ \\
\hline 1 & Activate their prior knowledge & 0 & 0 & $3(7.1)$ & $20(47.6)$ & $19(45.2)$ & $4.00(1)$ \\
\hline 2 & $\begin{array}{l}\text { Interpret, analyze, and apply key } \\
\text { concepts precisely }\end{array}$ & 0 & $3(7.1)$ & 0 & $21(50)$ & $18(42.9)$ & $4.00(1)$ \\
\hline 3 & Understand knowledge in-depth & $3(7.1)$ & $6(14.3)$ & 0 & $17(40.5)$ & $16(38.1)$ & $4.00(1)$ \\
\hline 4 & $\begin{array}{l}\text { Gain and retain knowledge for a long } \\
\text { period }\end{array}$ & $3(7.1)$ & $9(21.4)$ & 0 & $8(19)$ & $22(52.4)$ & $5.00(3)$ \\
\hline \multicolumn{2}{|c|}{ Development of Problem-solving skills } & $2(4.7)$ & $2(4.7)$ & $2(5.5)$ & $19(45.2)$ & 17 (39.6) & $\begin{array}{c}4.00 \\
(1.00)\end{array}$ \\
\hline 5 & Solve real-world problems & 0 & $6(14.3)$ & $2(4.8)$ & $17(40.5)$ & 17.40.5) & $4.00(1)$ \\
\hline 6 & $\begin{array}{l}\text { Consider alternatives when solving a } \\
\text { problem }\end{array}$ & $3(7.1)$ & 0 & $5(11.9)$ & $20(47.6)$ & $14(33.3)$ & $4.00(1)$ \\
\hline 7 & Make a reasonable conclusion & $3(7.1)$ & 0 & 0 & $20(47.6)$ & $19(45.2)$ & $4.00(1)$ \\
\hline \multicolumn{2}{|c|}{ Development of Self-directed learning } & $\mathbf{0}$ & $3(7.1)$ & $3(6.5)$ & $20(47.6)$ & $16(38.7)$ & $\begin{array}{l}4.00 \\
(1.00)\end{array}$ \\
\hline 8 & Identify their knowledge gaps & 0 & $3(7.1)$ & 0 & $23(54.8)$ & $16(38.1)$ & $4.00(1)$ \\
\hline 9 & Identify and use a variety of resources & 0 & $6(14.3)$ & $3(7.1)$ & $12(28.6)$ & $21(50)$ & $4.50(1)$ \\
\hline 10 & Think individually or by their own & 0 & 0 & $5(11.9)$ & $24(57.1)$ & $13(31)$ & $4.00(1)$ \\
\hline & Plan their work & 0 & $3(7.1)$ & $3(7.1)$ & $21(50)$ & $15(35.7)$ & $4.00(1)$ \\
\hline \multicolumn{2}{|c|}{ Improvement motivation } & $\mathbf{0}$ & $1.5(3.5)$ & $1.5(3.5)$ & $22(51.2)$ & $18(41.6)$ & $\begin{array}{l}4.00 \\
(1.00)\end{array}$ \\
\hline 12 & Stimulates students to learn more & 0 & $3(7.1)$ & 0 & $22(52.4)$ & $17(40.5)$ & $4.00(1)$ \\
\hline 13 & $\begin{array}{l}\text { Stimulates students' interest in } \\
\text { learning }\end{array}$ & 0 & 0 & $3(7.1)$ & $21(50)$ & $18(42.9)$ & $4.00(1)$ \\
\hline \multicolumn{2}{|c|}{ Promoting group collaboration } & $\mathbf{0}$ & $\mathbf{0}$ & $2(4.7)$ & $13(30.1)$ & $27(65)$ & $\begin{array}{l}5.00 \\
(1.00)\end{array}$ \\
\hline 14 & $\begin{array}{l}\text { Participate in an open discussion of } \\
\text { differing opinions }\end{array}$ & 0 & 0 & $3(7.1)$ & $11(26.2)$ & $28(66.7)$ & $5.00(1)$ \\
\hline 15 & $\begin{array}{l}\text { Share what they learned or } \\
\text { understood }\end{array}$ & 0 & 0 & $3(7.1)$ & $11(26.2)$ & $28(66.7)$ & $5.00(1)$ \\
\hline 16 & Accept and respect others opinion & 0 & 0 & 0 & $16(38.1)$ & $26(61.9)$ & $5.00(1)$ \\
\hline \multicolumn{2}{|c|}{$\begin{array}{l}\text { I prefer PBL to lecture as an effective } \\
\text { method of learning }\end{array}$} & $3(7.1)$ & $3(7.1)$ & $5(11.9)$ & $8(19)$ & 23(54.8) & $5.00(2.00)$ \\
\hline
\end{tabular}

$\mathrm{SDA}=$ Strongly Disagree, DA= Disagree, $\mathrm{N}=$ Neutral, $\mathrm{A}=$ Agree, $\mathrm{SA}=$ Strongly Agree, Mdn= Median, IQR= InterQuartile Range

In DTU, PBL assessment is a continuous assessment of students' performance during tutorials guided by a checklist with regard to content, process and professionalism. The assessment at the end of each session and midway during a module will be used as a formative assessment, while the end-of-module assessment will be used as summative assessment. However, in our study, half of the students did not think the assessment in PBL was appropriate, and about $42.2 \%$ of the students reported that it did not consider the improvements made by students with passage of time. Additionally, more than half of students $(53.1 \%)$ were satisfied with the overall implementation process of PBL (Table 4). 
Table 4: Students' assessment of PBL implementation process in the department of medicine and midwifery, DTU $(\mathrm{N}=308)$

\begin{tabular}{|c|c|c|c|c|c|c|c|}
\hline S.no & Question & $\begin{array}{l}\text { SDA } \\
\text { n(\%) }\end{array}$ & $\begin{array}{c}\text { DA } \\
\mathbf{n}(\%)\end{array}$ & $\begin{array}{c}\mathbf{N} \\
\mathbf{n}(\%)\end{array}$ & $\begin{array}{c}\mathbf{A} \\
\mathbf{n}(\%)\end{array}$ & $\begin{array}{c}\text { SA } \\
\mathbf{n}(\%)\end{array}$ & $\begin{array}{c}\text { Mdn } \\
\text { (IQR) }\end{array}$ \\
\hline \multicolumn{2}{|c|}{ Clear objectives/expectations } & $44(14.2)$ & 54(17.6) & 51(16.4) & $106(34.4)$ & 53(17.2) & $3.50(2.00)$ \\
\hline 1 & $\begin{array}{l}\text { Orientation on the PBL } \\
\text { tutorial process was provided } \\
\text { before the first session }\end{array}$ & $58(18.8)$ & $59(19.1)$ & $52(16.8)$ & $80(25.9)$ & $59(19.1)$ & $3.00(2)$ \\
\hline 2 & $\begin{array}{l}\text { The tutor made it clear right } \\
\text { from the start what they } \\
\text { expected from students in } \\
\text { PBL }\end{array}$ & $42(13.6)$ & $63(20.4)$ & $51(16.5)$ & 105(34) & $47(15.2)$ & $3.00(2)$ \\
\hline 3 & $\begin{array}{l}\text { The work expected or what to } \\
\text { do for PBL was known }\end{array}$ & $32(10.4)$ & $41(13.3)$ & $49(15.9)$ & 133(43) & $53(17.2)$ & $4.00(1)$ \\
\hline \multicolumn{2}{|c|}{$\begin{array}{l}\text { Cases and Composition of } \\
\text { modules }\end{array}$} & $19(6.2)$ & 37(12.0) & $50(16.3)$ & $141(45.7)$ & 61(19.7) & $4.00(1.00)$ \\
\hline 4 & $\begin{array}{l}\text { The contents of the PBL } \\
\text { cases were adjusted to your } \\
\text { level of learning or } \\
\text { understanding. }\end{array}$ & $32(10.4)$ & $41(13.3)$ & $27(8.7)$ & $143(46.3)$ & $65(21)$ & $4.00(1)$ \\
\hline 5 & $\begin{array}{l}\text { The PBL scenarios were } \\
\text { engaging and realistic. }\end{array}$ & $16(5.2)$ & 43(13.9) & $56(18.1)$ & $140(45.3)$ & $53(17.2)$ & $4.00(1)$ \\
\hline 6 & $\begin{array}{l}\text { The PBL cases integrated } \\
\text { knowledge across different } \\
\text { disciplines/courses. }\end{array}$ & $16(5.2)$ & 43(13.9) & $39(12.6)$ & $151(48.9)$ & $59(19.1)$ & $4.00(1)$ \\
\hline 7 & $\begin{array}{l}\text { The PBL cases foster } \\
\text { discussion at a higher level of } \\
\text { learning }\end{array}$ & $13(4.2)$ & $21(6.8)$ & $79(25.6)$ & $129(41.7)$ & $66(21.4)$ & $4.00(1)$ \\
\hline \multicolumn{2}{|c|}{ Workload } & 25(8.1) & $38(12.2)$ & 41(13.4) & $124(40.1)$ & $80(26.1)$ & $4.00(2.00)$ \\
\hline 8 & $\begin{array}{l}\text { The schedule of the PBL } \\
\text { sessions ( } 2 \text { meetings /week) } \\
\text { was adequate to address the } \\
\text { objectives. }\end{array}$ & $20(6.5)$ & $32(10.4)$ & $35(11.3)$ & $128(41.4)$ & $93(30.1)$ & $4.00(2)$ \\
\hline 9 & $\begin{array}{l}\text { The time given to each } \\
\text { session was enough to learn } \\
\text { during PBL sessions. }\end{array}$ & $8(2.6)$ & $30(9.7)$ & $24(7.8)$ & $144(46.6)$ & $102(33)$ & $4.00(1)$ \\
\hline 10 & $\begin{array}{l}\text { Overall, the workload } \\
\text { imposed by PBL was } \\
\text { reasonable. }\end{array}$ & $47(15.2)$ & $51(16.6)$ & $65(21)$ & $99(32)$ & $46(14.9)$ & $3.00(2)$ \\
\hline \multicolumn{2}{|c|}{ Assessment } & 57(18.4) & $60(19.4)$ & $71(23.2)$ & 89(29) & 31(10) & $3.00(2.00)$ \\
\hline 11 & $\begin{array}{l}\text { The tutors asked about } \\
\text { concepts and mechanisms } \\
\text { rather than facts. }\end{array}$ & $17(5.5)$ & $47(15.2)$ & $72(23.3)$ & $135(43.7)$ & $37(12)$ & $4.00(1)$ \\
\hline 12 & $\begin{array}{l}\text { The PBL evaluation was } \\
\text { both appropriate and fair. }\end{array}$ & $89(28.8)$ & $66(21.4)$ & $68(22)$ & $56(18.1)$ & $29(9.4)$ & $2.00(3)$ \\
\hline 13 & $\begin{array}{l}\text { It considers the } \\
\text { improvement made by the } \\
\text { students with time }\end{array}$ & $64(20.7)$ & $66(21.4)$ & 74(23.9) & $77(24.9)$ & $27(8.7)$ & $3.00(2)$ \\
\hline \multicolumn{2}{|c|}{ Satisfaction } & $\begin{array}{c}\text { VD } \\
\mathbf{n}(\%)\end{array}$ & $\begin{array}{c}\text { D } \\
\mathbf{n}(\%)\end{array}$ & $\begin{array}{c}\mathbf{U} \\
\mathbf{n}(\%)\end{array}$ & $\begin{array}{c}\mathbf{S} \\
\mathbf{n}(\%)\end{array}$ & $\begin{array}{c}\text { VS } \\
\mathbf{n}(\%)\end{array}$ & $\begin{array}{c}\text { Mdn } \\
\text { (IQR) }\end{array}$ \\
\hline 14 & $\begin{array}{l}\text { I am satisfied with the } \\
\text { process of PBL } \\
\text { implementation. }\end{array}$ & $36(11.7)$ & $54(17.5)$ & $54(17.5)$ & $132(42.7)$ & $32(10.4)$ & $4.00(2.00)$ \\
\hline
\end{tabular}


Table 5: Tutors' assessment of PBL implementation process in the department of medicine and midwifery, DTU $(\mathrm{N}=42)$.

\begin{tabular}{|c|c|c|c|c|c|c|c|}
\hline s.no & Question & $\begin{array}{l}\text { SDA } \\
\text { n(\%) }\end{array}$ & $\begin{array}{c}\text { DA } \\
\text { n(\%) }\end{array}$ & $\begin{array}{c}\mathbf{N} \\
\mathbf{n}(\%)\end{array}$ & $\begin{array}{c}\mathbf{A} \\
\mathbf{n}(\%)\end{array}$ & $\begin{array}{c}\text { SA } \\
\mathbf{n}(\%)\end{array}$ & $\begin{array}{l}\text { Mdn } \\
\text { (IQR) }\end{array}$ \\
\hline Clea & ar objectives/expectations & $\mathbf{0 ( 0 )}$ & 1(3.5) & $1.5(3.5)$ & 15(54.7) & 11(38.1) & $4.00(1.00)$ \\
\hline 1 & $\begin{array}{l}\text { I know what to do or what is } \\
\text { expected from me for } \\
\text { implementing PBL }\end{array}$ & 0 & 0 & $3(7.1)$ & $22(52.4)$ & $17(40.5)$ & $4.00(1)$ \\
\hline 2 & $\begin{array}{l}\text { I make it clear right from the } \\
\text { start what is expected from } \\
\text { students in PBL tutorial }\end{array}$ & 0 & $3(7.1)$ & 0 & $24(57.1)$ & $15(35.7)$ & $4.00(1)$ \\
\hline \multicolumn{2}{|c|}{$\begin{array}{l}\text { PBL Cases and Composition of } \\
\text { modules }\end{array}$} & $4(10.1)$ & $5(11.9)$ & $6(13.7)$ & $19(44)$ & $8(20.2)$ & $4.00(1.00)$ \\
\hline 3 & $\begin{array}{l}\text { The contents of the PBL cases } \\
\text { were adjusted to students' level } \\
\text { of learning or understanding. }\end{array}$ & $11(26.2)$ & $7(16.7)$ & $7(16.7)$ & $15(35.7)$ & $2(4.8)$ & $3.00(3)$ \\
\hline 4 & $\begin{array}{l}\text { The PBL scenarios were } \\
\text { engaging and realistic. }\end{array}$ & $3(7.1)$ & $3(7.1)$ & $9(21.4)$ & $25(59.5)$ & $2(4.8)$ & $4.00(1)$ \\
\hline 5 & $\begin{array}{l}\text { The PBL cases integrated } \\
\text { knowledge across different } \\
\text { disciplines/courses. }\end{array}$ & $3(7.1)$ & $7(16.7)$ & $4(9.5)$ & $17(40.5)$ & $11(26.2)$ & $4.00(2)$ \\
\hline 6 & $\begin{array}{l}\text { The PBL cases foster discussion } \\
\text { at a higher level of learning }\end{array}$ & 0 & $3(7.1)$ & $3(7.1)$ & $17(40.5)$ & $19(45.2)$ & $4.00(1)$ \\
\hline \multicolumn{2}{|c|}{ Workload } & $1(2.4)$ & $9(23)$ & $5(11)$ & $18(43)$ & 9(20.6) & $4.00(1.25)$ \\
\hline 7 & $\begin{array}{l}\text { The schedule of the PBL } \\
\text { sessions ( } 2 \text { meetings /week) } \\
\text { was adequate to address the } \\
\text { objectives. }\end{array}$ & $3(7.1)$ & $9(21.4)$ & $2(4.8)$ & $18(42.9)$ & $10(23.8)$ & $4.00(2)$ \\
\hline 8 & $\begin{array}{l}\text { The time given to each session } \\
\text { ( } 2 \text { hours/session) was enough } \\
\text { to learn during PBL sessions. }\end{array}$ & 0 & $11(26.2)$ & 0 & $21(50.0)$ & $10(23.8)$ & $4.00(2)$ \\
\hline 9 & $\begin{array}{l}\text { Overall, the workload imposed } \\
\text { by PBL was reasonable. }\end{array}$ & 0 & $9(21.4)$ & $12(28.6)$ & $15(35.7)$ & $6(14.3)$ & $3.50(1)$ \\
\hline \multicolumn{2}{|r|}{ Assessment } & $1(2.4)$ & $1(2.4)$ & $3(7.1)$ & 20(47.6) & $17(40.5)$ & $4.00(1.00)$ \\
\hline 10 & $\begin{array}{l}\text { I provided constructive } \\
\text { feedback timely. }\end{array}$ & $3(7.1)$ & 0 & 0 & $22(52.4)$ & $17(40.5)$ & $4.00(1)$ \\
\hline 11 & $\begin{array}{l}\text { The PBL evaluation was both } \\
\text { appropriate and fair. }\end{array}$ & 0 & 0 & $6(14.3)$ & $18(42.9)$ & $18(42.9)$ & $4.00(1)$ \\
\hline 12 & $\begin{array}{l}\text { The PBL assessment method } \\
\text { considers the improvement } \\
\text { made by the students with } \\
\text { time }\end{array}$ & 0 & $3(7.1)$ & $3(7.1)$ & $20(47.6)$ & $16(38.1)$ & $4.00(1)$ \\
\hline \multicolumn{2}{|c|}{ Satisfaction } & $\begin{array}{l}\text { VD } \\
\mathbf{n}(\%)\end{array}$ & $\begin{array}{c}D \\
\mathbf{n}(\%)\end{array}$ & $\begin{array}{c}\mathrm{U} \\
\mathbf{n}(\%)\end{array}$ & $\begin{array}{c}\mathbf{S} \\
\mathbf{n}(\%)\end{array}$ & $\begin{array}{c}\text { VS } \\
\mathbf{n}(\%)\end{array}$ & $\begin{array}{l}\text { Mdn } \\
\text { (IQR) }\end{array}$ \\
\hline 13 & $\begin{array}{l}\text { I am satisfied with the process } \\
\text { of PBL implementation. }\end{array}$ & $3(7.1)$ & $6(14.3)$ & $3(7.1)$ & $15(35.7)$ & $15(35.7)$ & $4.00(2.00)$ \\
\hline
\end{tabular}

$\mathrm{SDA}=$ Strongly Disagree, $\mathrm{DA}=$ Disagree, $\mathrm{N}=$ Neutral, $\mathrm{A}=$ Agree, $\mathrm{SA}=$ Strongly Agree, $\mathrm{Mdn}=$ Median, $\mathrm{IQR}=$ Inter-Quartile Range, $\mathrm{VD}=$ Very Dissatisfied, $\mathrm{D}=$ Dissatisfied, $\mathrm{U}=$ Unsatisfied, $\mathrm{S}=$ Satisfied, VS= Very Satisfied 
Tutors rated the implementation process of PBL more favorably than students. Unlike students, the top two tutors' agreements were on the existence of clear expectations or what to do in PBL sessions $(92.8 \%)$ and the appropriateness of PBL assessment. Similar to students, most tutors considered the workload imposed by PBL was reasonable $(63.6 \%)$, and $\mathrm{PBL}$ cases were appropriate $(64.2 \%)$. Additionally, tutors' satisfaction on the overall implementation process of PBL was higher than students' rating (71.4\%) (Table 5).

Academic leaders' response on the appropriateness of $\mathrm{PBL}$ implementation and cases was consistent with students' and tutors' ratings. The key informant interviews demonstrated the preparation and hardwork put into the development of quality cases. They said that more than 350 PBL cases had been constructed and revised and the Vice Dean said:

"I believe most of PBL cases were in line with the principles of case development. The HSEDC [Health Science Education Development Centre] office tried to improve cases by forming a multi-disciplinary team even though a few cases need more integration."

Additionally, interviewed leaders thought the implementation was in line with the principles of PBL, and working on its sustainability was the priority. Midwifery department head mentioned:

'PBL is implemented successfully even if some little gaps happen as we are new for $P B L$. So I believe these gaps are not major factors that compromise its effective implementation.'

Students and tutors performance throughout PBL sessions: Students witnessed the contribution of tutors in enabling them to attain the desired competencies: promoting constructive/active learning skill (71.3\%), contextual learning skill (67\%) and self-directed learning skill $(64.2 \%)$ were the top three competencies facilitated by tutors during PBL sessions. However, only $37.6 \%$ of students agreed on the intra-personal behaviour of tutors in terms of a motivation to fulfil their role and having a clear picture of his/her strengths and weakness (Table 6). Most tutors for their part agreed that students demonstrated the desired learning outcomes: constructive/active learning skills (87.5\%), critical appraisal skills $(81 \%)$ and collaborative learning skills $(78.6 \%)$ were the top three learning skills demonstrated by students during PBL sessions.

Factors that facilitate PBL implementation: Key informant interviews with academic leaders identified factors that facilitate PBL implementation related to students, tutors, curriculum, institutional (infrastructure and human resource) and programmatic issues (coordination and monitoring).

Students and tutors: Students' and tutors' acceptance of PBL and a strong commitment to their roles, particularly tutors' willingness to be engaged in case preparation and review, were the major opportunities for effective implementation. The Vice Dean of the college reported:

"It is obvious that students were committed to share what they know, discuss together, provide constructive feedback and prepare adequately in a learning issue constructed. This contributed highly for effective implementation of PBL."

Curriculum: Respondents acknowledged the role of the curriculum in providing a contractual agreement between tutors, students and institutions. They emphasized the contribution of the curriculum in integrating different disciplines and providing the type of cases for all weeks. The following extract from social and public health unit coordinator yielded:

"The curriculum has a great contribution for running PBL as a guiding frame in integrating and providing cases as well as schedules by adjusting the compositions of modules with students level of learning."

Infrastructure: Respondents described that the college provided adequate resources like small group rooms, charts, markers and whiteboards. A biomedical unit coordinator said:

"I think the college provides adequate resources for PBL implementation. But still internet service is scarce." 
Table 6: Students' evaluation of tutors performance throughout PBL sessions in the department of medicine and midwifery, DTU, $2018(\mathrm{~N}=308)$.

\begin{tabular}{|c|c|c|c|c|c|c|c|}
\hline s.no & Items & $\begin{array}{l}\text { SDA } \\
\text { n(\%) }\end{array}$ & $\begin{array}{c}\text { DA } \\
\text { n(\%) }\end{array}$ & $\begin{array}{c}\mathbf{N} \\
\mathbf{n}(\%)\end{array}$ & $\begin{array}{c}\mathbf{A} \\
\mathbf{n}(\%)\end{array}$ & $\begin{array}{c}\text { SA } \\
\text { n(\%) }\end{array}$ & $\begin{array}{c}\text { Mdn } \\
\text { (IQR) }\end{array}$ \\
\hline \multicolumn{2}{|c|}{ Constructive/active learning } & $14(4.5)$ & $29(9.4)$ & $45(14.7)$ & $153(49.7)$ & 67(21.6) & $4.00(1.00)$ \\
\hline 1 & $\begin{array}{l}\text { Encourage students to } \\
\text { express their own opinions }\end{array}$ & $23(7.4)$ & $30(9.7)$ & $38(12.3)$ & $161(52.1)$ & $56(18.1)$ & $4.00(1)$ \\
\hline 2 & $\begin{array}{l}\text { Encourage students to } \\
\text { understand underlying } \\
\text { mechanisms/theories }\end{array}$ & 11(3.6) & $30(9.7)$ & $58(18.8)$ & $144(46.6)$ & $65(21)$ & $4.00(1)$ \\
\hline 3 & $\begin{array}{l}\text { Invite students to provide } \\
\text { the reasoning behind their } \\
\text { opinions }\end{array}$ & $8(2.6)$ & $27(8.7)$ & $40(12.9)$ & $154(49.8)$ & $79(25.6)$ & $4.00(1)$ \\
\hline \multicolumn{2}{|c|}{ Self-directed learning } & $16(5.3)$ & 34(11.1) & $60(19.4)$ & $142(46.1)$ & $56(18.1)$ & $4.00(1.00)$ \\
\hline 4 & $\begin{array}{l}\text { Encourage students to } \\
\text { generate clear learning } \\
\text { issues by themselves }\end{array}$ & $22(7.1)$ & $49(15.9)$ & $54(17.5)$ & $123(39.8)$ & $60(19.4)$ & $4.00(1)$ \\
\hline 5 & $\begin{array}{l}\text { Encourage students to } \\
\text { search for various } \\
\text { resources by themselves }\end{array}$ & $12(3.9)$ & $20(6.5)$ & $57(18.4)$ & $156(50.5)$ & $63(20.4)$ & $4.00(1)$ \\
\hline 6 & $\begin{array}{l}\text { Invite students to discuss } \\
\text { by guiding from a side } \\
\text { rather than giving you the } \\
\text { answer }\end{array}$ & $15(4.9)$ & $34(11)$ & $68(22)$ & $147(47.6)$ & $44(14.2)$ & $4.00(1)$ \\
\hline \multicolumn{2}{|c|}{ Contextual learning } & 11(3.6) & $29(9.4)$ & $62(20)$ & 154(50.1) & $52(16.9)$ & $4.00(1.00)$ \\
\hline 7 & $\begin{array}{l}\text { Support students to apply } \\
\text { knowledge on the given } \\
\text { problem }\end{array}$ & $14(4.5)$ & $28(9.1)$ & $47(15.2)$ & $165(53.4)$ & $54(17.5)$ & $4.00(1)$ \\
\hline 8 & $\begin{array}{l}\text { Support students to apply } \\
\text { knowledge to other } \\
\text { situations/problems }\end{array}$ & $8(2.6)$ & $30(9.7)$ & $76(24.6)$ & 144(46.6) & $50(16.2)$ & $4.00(1)$ \\
\hline \multicolumn{2}{|c|}{ Collaborative learning } & 27(8.6) & $42(13.5)$ & $68(22.1)$ & 133(43.1) & $38(12.6)$ & $4.00(1.00)$ \\
\hline 9 & $\begin{array}{l}\text { Provide constructive } \\
\text { feedback about your group } \\
\text { work }\end{array}$ & $30(9.7)$ & $41(13.3)$ & $71(23)$ & $127(41.1)$ & $39(12.6)$ & $4.00(1)$ \\
\hline 10 & $\begin{array}{l}\text { Provide constructive } \\
\text { feedback about your } \\
\text { contribution }\end{array}$ & $36(11.7)$ & $56(18.1)$ & $67(21.7)$ & $122(39.5)$ & $27(8.5)$ & $3.00(2)$ \\
\hline 11 & $\begin{array}{l}\text { Invite students to } \\
\text { participate in discussion } \\
\text { more evenly }\end{array}$ & $14(4.5)$ & $28(9.1)$ & $66(21.4)$ & $149(48.2)$ & $51(16.5)$ & $4.00(1)$ \\
\hline \multicolumn{2}{|c|}{$\begin{array}{l}\text { Intra-personal behavior as a } \\
\text { tutor }\end{array}$} & 57(18.5) & $65(21.1)$ & $70(22.7)$ & $86(27.9)$ & $30(9.7)$ & $3.00(2.00)$ \\
\hline 12 & $\begin{array}{l}\text { Has a clear picture of } \\
\text { his/her strengths and } \\
\text { weaknesses }\end{array}$ & $64(20.7)$ & $72(23.3)$ & $69(22.3)$ & $71(23)$ & $32(10.4)$ & $3.00(2)$ \\
\hline 13 & $\begin{array}{l}\text { Motivated to fulfill his/her } \\
\text { role as a tutor }\end{array}$ & $50(16.2)$ & $58(18.8)$ & $71(23)$ & $101(32.7)$ & $28(9.1)$ & $3.00(2)$ \\
\hline
\end{tabular}

$\mathrm{SDA}=$ Strongly Disagree, DA= Disagree, $\mathrm{N}=$ Neutral, A= Agree, SA= Strongly Agree, Mdn= Median, IQR= InterQuartile Range 
Human resource: Hiring more tutors has been a priority, with more than 250 instructors employed by the college. Furthermore, PBL implementation started after providing training for the existing instructors and continued for newly employed staff. Since the introduction of PBL, almost all (250 employed academic staff) have been trained. The college Vice Dean reflects the same:

“... almost all newly hired staff have been trained in PBL before involving them in case preparation and tutoring which contributes more for its effective implementation."

Coordination: Smooth coordination starts within the department where department heads played the role of assigning tutors and providing readyprinted cases. PBL coordinators at the department and college level oversee the implementation. That being said, coordinating multidisciplinary teams for case review and development needs to be strengthened.

Monitoring: The college established an office called Health Science Educational Development Centre (HSEDC) mainly responsible for assuring quality education by identifying gaps and intervening in collaboration with departments and the college. The following extract from the college Vice Dean indicates:

"HSEDC is responsible for assuring the quality of cases and implementation process...tutoring. Additionally, there is a $P B L$ committee selected from all departments under HSEDC structure that identified gaps and provided interventions accordingly."

Factors that impede PBL implementation: Even though all interviewed key informants did not mention any barrier, most students listed heavy workload $(73.1 \%)$ and insufficient time for self-study $(67.6 \%)$ as major barriers in the selfadministered questionnaire. Most tutors for their part described inadequate incentive $(81 \%)$ and frequent change of leadership $(57.1 \%)$ in the college as major barriers for PBL implementation.

\section{DISCUSSION}

This study provides the first local evidence on PBL from the perspective of students, tutors and academic leaders. Students, tutors and academic leaders evaluated PBL favorably. It was deemed effective in promoting student learning. Its implementation was considered consistent with the principles of PBL. Students rated tutors' performance positively, and tutors also rated student learning affirmatively. Respondents identified facilitating factors related to students, tutors, curriculum, infrastructure, human resources and program coordination and management. The major barriers were heavy workload and insufficient time for self-study from students' standpoint and lack of incentives and frequent leadership turnover from tutors standpoint.

In our study, students, tutors and academic leaders preferred PBL and perceived it as an effective teaching method in facilitating students' learning in terms of knowledge construction, developing problem-solving and self-directed learning skills, enhancing group collaboration and improving students' motivation to learn. Different studies conducted among students $(12,13,16)$ and tutors $(17,18)$ reported similar results on the perceived effectiveness of PBL.

In this study, the implementation process of PBL was characterized by the existence of clear objectives/expectations, appropriate PBL cases, and reasonable workload. Studies in Korea (19), Saudi (13) and Egypt (20) have reported students' agreement with appropriateness of problem scenarios and the workload. However, students did not think PBL assessment was appropriate which could be related to the absence of formative assessment that consider the improvement of students' performance through time. Another study in Saudi has also reported students' displeasure with the PBL assessment (14). Despite the fact that students should know the work expected or what to do in PBL session, almost half of the students disagreed on the existence of clear objective/expectation. This implied that adequate orientation on PBL tutorial process should be given for students before their exposure to the first session and tutors are 
expected to make clear what is expected from students in PBL tutorials.

Additionally, in our study, students rated tutors' performance positively and tutors also rated student learning affirmatively. However, only $37.6 \%$ of the students agreed on the intrapersonal behavior of tutors characterized by understanding his/her strengths and weakness. This indicated that tutors should develop a habit of providing and receiving feedback at the end of PBL sessions.

In our study, the factors that facilitate PBL implementation were students' and tutors' preferences and commitment to their roles, the contribution of the curriculum in integrating different disciplines and providing the content and schedule of PBL, and institutional leadership ensuring provision of adequate infrastructure, hiring more faculty members, continuous faculty development, strong coordination and the existence of monitoring mechanism. This was consistent with a study done in Malawi (21) and implementation of PBL at a medical school in Ghana faced challenges arising from curriculum design, resource limitations, tutors and case scenarios (22).

From the students' side, heavy workload and insufficient time for self-study and from tutors' side lack of incentives and frequent leadership turnover were the major barriers of PBL implementation. These views are in agreement with results of other studies conducted among students in Egypt (11) and tutors in Nigeria (23).

One important limitation of this study is that we did not evaluate attainment of the desired learning outcomes objectively to speak more confidently about the effectiveness of PBL in improving student learning. The other weakness is that our study is limited to one institution and may not be generalized to other institutions.

In conclusion, our study supports the introduction of problem-based learning in Ethiopia. Students, tutors and academic leaders liked PBL and consider it an effective teaching method. They were satisfied with the overall implementation process and acknowledged the presence of enabling conditions for a successful implementation. However, improving the PBL assessment method and the training or orientation given before starting the PBL sessions have to be strengthened. We recommend that future studies to objectively evaluate attainment of student learning outcomes.

\section{ACKNOWLEDGMENTS}

The authors thank all participants in the College of Health Sciences, Debre Tabor University, who accepted to be part of this study.

\section{REFERENCES}

1. Schmidt HG, Rotgans JI \& Yew EH. The process of problem-based learning: what works and why. Medical Education. 2011; 45: 792-806. doi:10.1111/j.13652923.2011.04035.x

2. Taylor D, Miflin B. Problem-based learning: Where are we now? Medical Teacher. 2008; 30 (8):742 -763. DOI: 10.1080/01421590802217199

3. Gerald $\mathrm{C}-\mathrm{H}$ Koh. Revisiting the 'Essentials of problem-based learning'. Medical Education 2016: 50: 596-599, doi: 10.1111/medu.12794

4. Gerald C-H Koh, Khoo HE, Wong ML, Koh D. The effects of problem-based learning during medical school on physician competency: a systematic review. CMAJ 2008;178(1):34-41. DOI:10.1503/cmaj.070565

5. Barrows, H.S. \& Tamblyn, R.M. (1980), Problem-based Learning, an approach to medical education. Springer Publishing, New York.

6. Patrick Lai, Obstacles to the implementation of problem-based learning (PBL) in Local Universities of Hong Kong. The Hong Kong Institute of Education, Hong Kong, SAR, China

7. Yodit Abraham, Aklilu Azaje. The new innovative medical education system in Ethiopia: Background and development. Ethiop. J. Health Dev. 2013;27 Special Issue 1:36-40. 
8. Debre Tabor University. A hybrid innovative medical curriculum. 2013

9. Amoako-Sakyi D and Amonoo-Kofi H. Problem-based learning in resource-poor settings: lessons from a medical school in Ghana. BMC Medical Education (2015) 15:22. DOI 10.1186/s12909-015-0501-4

10. Vardi, Iris and Ciccarelli, Marina. Overcoming

problems in problem-based learning: a trial of strategies in an undergraduate unit. Innovations in Education and Teach ing International. 45 (4): pp. 345-354.

11. Sahar Younes and Samah Anwar. Students' Perception and Acceptance of Problem-based Learning Approach in Critical Care Nursing Practice. Scientific Cooperations International Workshops on Medical Topics, Ankara-TURKEY, 7-8 June 2014

12. Abdulmajeed A. Al-Drees, Mahmoud S. Khalil, Mohammad Irshad, and Hamza M. Abdulghani. Students' perception of the problem-based learning tutorial session in a system-based hybrid curriculum. Saudi Med J 2015; Vol. 36 (3)

13. Bader Shamsan and A.T. Syed. Evaluation of Problem Based Learning Course at the College of Medicine, Qassim University, Saudi Arabia. Al-Melinda - 51442

14. Min Jeong Kim. Students' Satisfaction and Perception of Problem Based Learning Evaluated by Questionnaire. Kosin Medical Journal 2015;30:149-157.

15. Braun, V., \& Clarke, V. (2006). Using thematic analysis in psychology. Qualitative Research in Psychology, 3, 77-101.
16. Shahid S. Alkhuwaiter, Roqayah I. Aljuailan, Saeed M. Banabilh. Problembased learning: Dental student's perception of their educational environments at Qassim University. Journal of International Society of Preventive and Community Dentistry. http://www.jispcd.org on Tuesday, May 29, 2018, IP: 197.156.95.216

17. Joseph Kigen Katwa, Ronald Omenge Obwoge, Joice Baliddawa, Laban Ayiri, Robert Kei. Knowledge and Perception of Lecturers regarding Problem- Based Learning as an Educational Approach in College of Health Sciences, Moi University. IOSR Journal of Research \& Method in Education (IOSR-JRME) eISSN: 2320-7388,p-ISSN: 2320-737X Volume 8, Issue 1 Ver. IV (Jan. - Feb. 2018), PP 26-29

18. Moses Galukande, Achilles Katamba \& Nelson Sewankambo. Problem-based learning: tutors' views 5 years after implementation at a sub-Saharan University. Afr Health Sci. 2015 Mar; 15(1): 261-268

19. Ghadeer Al-Shaikh, Eman M Al Mussaed, Tahani N Altamimi, HalaElmorshedy, Sadiqa Syed, Farida Habib. Perception of Medical Students Regarding Problem Based Learning.Kuwait Medical Journal 2015; 47 (2): 134 - 139.

20. Miller, S.K. (2003). A comparison of students' outcomes following problembased learning instruction versus traditional lecture learning in a graduate pharmacology course. Journal of American Academic Practice, 15, 550556. 
21. Giva, K.R.N. \& Duma, S.E., 2015, 'Characteristics and critical success factors for implementing problem-based learning in a human resource-constrained country', Curationis 38(1), Art. \#1283, 11 pages. http://dx.doi.org/10.4102/ curationis.v38i1.1283

22. Amoako-Sakyi and Amonoo-Kuofi. Problem-based learning in resource-poor settings: lessons from a medical school in Ghana. BMC Medical Education (2015) 15:221 DOI 10.1186/s12909-015-0501-4

23. Al-Naggar, R.A., \& Bobryshev, Y.V. (2012). Acceptance of Problem Based Learning among Medical Students. $J$ Community Med Health Educ, 2:146.doi:10.4172/2161-0711.1000146. 